\title{
DISTORSIONES FISCALES Y LA CURVA DE LAFFER EN LA REPÚBLICA DOMINICANA
}

\section{Fiscal Distortions and the Laffer Curve in the Dominican Republic}

\section{Peter Albert Prazmowski}

Grupo para la Productividad y Competitividad Nacional (GPCN) y E. León Jimenes, República Dominicana

Correo-e: peterp@gpcn.org.do

https://orcid.org/0000-0001-8590-003X

Recibido: 7/10/2019 • Aprobado: 7/2/2020

Cómo citar: Prazmowski, P. A. (2020). Distorsiones fiscales y la curva de Laffer en la República Dominicana. Ciencia y Sociedad, 45(1), 7-23. Doi: https://doi.org/10.22206/cys.2020.v45i1.pp7-23

\section{Resumen}

Este artículo estima una curva de Laffer para la República Dominicana utilizando datos anuales desde el año 1955 hasta el 2018. Los resultados indican que la presión tributaria que maximizaría las recaudaciones fiscales es de aproximadamente un $20 \%$, superior al promedio histórico de $15 \%$. La baja presión tributaria es en parte el resultado de distorsiones fiscales que incluyen evasión, elución e informalidad. Se estima que dichas distorsiones son de aproximadamente un $60 \%$ y su evolución histórica se corresponde con numerosos episodios, incluyendo más de veinte reformas tributarias y cinco acuerdos de estabilización con el Fondo Monetario Internacional (FMI). Las conclusiones pueden ser útiles para el diseño e implementación de políticas fiscales en general y tributarias en particular.

Palabras claves: curva de Laffer; República Dominicana; política fiscal; tributación; impuestos.

\begin{abstract}
This paper estimates a Laffer curve for the Dominican Republic using annual data from 1955 to 2018. The results indicate that the tax burden that would maximize tax revenues is approximately $20 \%$, higher than the historical average of $15 \%$. The low tax burden is, in part, the result of tax distortions that include evasion, avoidance, and informality. It is estimated that these distortions are approximately $60 \%$, and their historical evolution corresponds to numerous episodes, including more than 20 tax reforms and five stabilization agreements with the International Monetary Fund (IMF). The conclusions can be useful for the design and implementation of fiscal policies in general and tax policies in particular.
\end{abstract}

Keywords: Laffer curve; Dominican Republic; fiscal policy; taxation; taxes.

Clasificación del JEL: C5; E6; H2; H3. 


\section{Introducción}

La curva de Laffer ilustra una relación teórica entre los ingresos fiscales y la presión tributaria. Desde su introducción en 1974, durante la administración del Presidente Norteamericano Gerard Ford, la curva de Laffer ha inspirado un programa de investigación que redefinió globalmente el criterio y alcance de la política fiscal (ver, por ejemplo, Blundell, 1992 y más recientemente a Canto, Joines y Laffer, 2014, para una revisión de la literatura). Una de las implicaciones derivadas de la curva de Laffer, es que un aumento de las tasas impositivas, más allá de cierto punto máximo denominado el óptimo de Laffer, podría causar una caída en los ingresos fiscales. ${ }^{1}$ Esta caída es el resultado de la interacción entre un efecto aritmético y un efecto económico sobre el comportamiento de los contribuyentes.

El efecto aritmético muestra el cambio lineal en los ingresos fiscales de aplicar una tasa impositiva diferente sobre la misma base imponible. El efecto económico mide los cambios en el comportamiento de los contribuyentes ante diferentes niveles de impuestos. Por ejemplo, a medida que aumentan los impuestos, las empresas y los agentes económicos tendrán menos incentivos para producir y trabajar. Por ende, mientras mayores son los impuestos, mayor será el efecto económico de la tributación, hasta el punto donde se supera el efecto aritmético, causando una caída en los ingresos fiscales. Esto implica que, si la economía está en el lado descendente de la curva de Laffer, el gobierno puede, en principio, aumentar los ingresos tributarios si reduce los impuestos.

1. El óptimo de Laffer es diferente al óptimo que se encuentra, por ejemplo, en la literatura sobre crecimiento económico. Según Laffer, la presión fiscal óptima es aquella que maximiza las recaudaciones del gobierno. Según la literatura sobre el crecimiento económico, la presión fiscal óptima es aquella que maximiza el crecimiento presente y futuro de la economía, la cual es diferente y probablemente inferior a la sugerida por la curva de Laffer (Barro y Sala-i-Martin, 1992).
Aunque el concepto Laffer ha sido malinterpretado, como lo indica Blinder (1981), el mismo proporciona una aproximación del nivel de impuestos que maximiza los ingresos fiscales del gobierno y, por lo tanto, qué tan distante está un país de dicho nivel. La forma exacta de la curva de Laffer es desconocida y solo puede estimarse empíricamente (Gahvari, 1989). En adición, y debido a las complejidades del sistema tributario, estimar la curva de Laffer es una tarea desafiante (Henderson, 1981). No obstante, el ejercicio de estimar una curva de Laffer podría proporcionar información relevante sobre la naturaleza y efectividad del sistema tributario de un país.

Existen muchas versiones y agregaciones que permiten estimar empíricamente la curva de Laffer, sin comprometer conclusiones útiles y relevantes. Por ejemplo, como en Trabandt y Uhlig (2013), la curva de Laffer podría utilizarse para estimar la presión tributaria óptima y compararlo con la presión tributaria entre diferentes economías. Estas comparaciones son útiles para identificar las características específicas de los sistemas tributarios y derivar lecciones relevantes de política tributaria.

Este artículo estima una curva de Laffer para la República Dominicana (RD), empleando datos anuales que van desde 1955 hasta 2018. Uno de los objetivos del ejercicio es determinar dónde se encuentra el país en la curva y estimar la presión tributaria que maximizaría las recaudaciones del gobierno dominicano. Los resultados confirman la relevancia empírica de la curva de Laffer en la República Dominicana, e indican que la presión tributaria que maximizaría los ingresos fiscales es de alrededor del $20 \%$. Esta presión es significativamente superior al promedio de $15 \%$ que ha prevalecido en el histórico del país.

A pesar de las numerosas reformas tributarias que ha implementado la República Dominicana desde 
la década de los años cincuenta, es indudable lo difícil que ha sido aumentar la presión tributaria. Este documento muestra que existen distorsiones en el sistema tributario dominicano que han impedido aumentar la presión tributaria por encima de un techo histórico de $18 \%$. En particular, la evasión, la elusión y la informalidad en el sistema productivo nacional son factores que explican el fracaso de las reformas tributarias implementadas, y la razón por la cual, la República Dominicana es uno de los países de América Latina y el Caribe, con menor presión tributaria.

La literatura ha investigado empíricamente los efectos e implicaciones de la evasión, elusión e informalidad sobre el tamaño y la forma de la curva de Laffer (Sanyal, Gang y Goswami, 2000). Sin embargo, el alcance de esta literatura es limitado, a pesar del fracaso de las reformas fiscales implementadas en la mayoría de los países en vías de desarrollo, para aumentar la presión tributaria y ayudar a equilibrar los déficits fiscales. ${ }^{2}$

Este documento introduce formalmente las distorsiones fiscales en el modelo de la curva de Laffer y muestra que, ante la presencia de evasión, elusión e informalidad, la presión tributaria se ve socavada ya que la forma y tamańo de la curva se mueve con dichas distorsiones. En contraste con Busato y Chiarini (2013), donde las distorsiones fiscales cambian la altura de la curva de Laffer, el enfoque en el presente documento muestra que las distorsiones fiscales cambian el efecto económico de los impuestos, haciendo que la curva de Laffer se reduzca tanto en altura como en longitud. ${ }^{3}$

2. Estudios empíricos incluyen a Feige y McGee (1983), van Ravestein y Vijlbrief (1988), Hsing (1996), Heijman y van Ophem (2005).

3. Intuitivamente, las distorsiones fiscales hacen que los ingresos fiscales sean más sensibles al efecto económico de los impuestos. Esta sensibilidad reduce la base y el alcance de los impuestos sobre los ingresos tributarios (Friedman, Johnson, Kaufmann y Zoido-Lobaton, 2000).
Este método permite estimar el nivel de distorsiones presentes en una economía. En particular, las desviaciones en la curva de Laffer producto de las distorsiones fiscales, se pueden utilizar para aproximar empíricamente la magnitud de dichas distorsiones. Esto se logra incorporando los conceptos de ingresos tributarios "observados" y "no observados" que resultan de dichas distorsiones. Además, la técnica de estimación de los componentes "observados" y "no observados", permite medir la evolución de las distorsiones en el tiempo, arrojando conclusiones interesantes sobre la efectividad de las reformas tributarias que fueron implementadas históricamente en la República Dominicana.

El ejercicio estima, que en promedio, el tamaño combinado de la evasión, elusión e informalidad en la República Dominicana es de aproximadamente un $60 \%$. Este es el nivel de distorsiones que debe ser eliminado para que la curva de Laffer dominicana converja a la que es teóricamente ideal; es decir, aquella donde las recaudaciones desaparecen cuando la prisión fiscal llega al 100 \%. En adición, el análisis muestra como han reaccionado y evolucionado históricamente la evasión, elusión e informalidad ante las diferentes reformas tributarias que el país ha implementado.

Esta investigación es relevante ya que la República Dominicana es un país con una historia fascinante que va desde un régimen dictatorial, que culmina a principios de la década de los años sesenta, hacia una democracia joven, la cual ha tenido varios episodios de crisis y estabilización económica. Además, el país ha tenido históricamente una reputación de instituciones débiles, sujetas a corrupción e impunidad, las cuales han permitido, en parte, los altos niveles de evasión, elusión e informalidad observados. Finalmente, la forma propuesta de estimar empíricamente dichas distorsiones es novedosa y podría replicarse en otras economías.

En lo que resta del artículo, la sección 2 presenta una visión general de la República Dominicana y 
su postura fiscal desde la década de los años sesenta. La sección 3 muestra los fundamentos teóricos de la curva de Laffer e introduce formalmente el concepto de distorsiones fiscales en el modelo. La sección 4 presenta el ejercicio empírico y realiza estimaciones de la curva de Laffer para la República Dominicana. La sección 5 estima las distorsiones fiscales y su conexión con las políticas fiscales implementadas históricamente en el país. La sección 6, concluye.

\section{Impuestos en la República Dominicana}

Antes de entrar en los fundamentos teóricos y realizar el ejercicio empírico para estimar la curva de Laffer, esta sección presenta una descripción histórica de los impuestos en la República Dominicana y su conexión con los episodios macroeconómicos más relevantes del país. La figura 1 muestra la evolución de los ingresos tributarios y del PIB, en términos reales per cápita, para el período comprendido entre 1955 y $2018 .^{4}$

Claramente, los ingresos fiscales y el PIB se han movido proporcionalmente. Sin embargo, los ingresos fiscales muestran mayor volatilidad que el PIB, sobre todo en tiempos de crisis. Hay una caída significativa en 1965 durante el período de transición entre la decadencia del régimen dictatorial de Rafael Leonidas Trujillo, que termina en 1962, y el establecimiento de un nuevo gobierno democrático en 1965 . Al borde de una crisis social y política, estalló una guerra civil el 24 de abril de 1965 , cuando el Presidente Johnson de los Estados Unidos de Norteamérica, ordenó la intervención de sus tropas militares en el país. El conflicto duró poco; en 1966 se promulgó una nueva constitución y se celebraron elecciones, donde se nombró a Joaquín Balaguer como el primer presidente constitucional post-trujillista. Claramente, la capacidad de recaudar impuestos en un entorno inestable era difícil y los

4. La data proviene del FMI International Financial Statistics. ingresos tributarios se volvieron más sensibles a la volatilidad.

El nuevo gobierno y las políticas de transición hacia la democracia estimularon la inversión privada, al mismo tiempo que aumentaron la producción y los ingresos tributarios. Esta tendencia continuó hasta 1975, cuando producto de elevados niveles de deuda, se produjo una crisis económica que empujó al país a la peor y más larga recesión en su historia. La disminución en la producción y en los ingresos tributarios continuaron hasta 1982, cuando un cambio de gobierno y un programa de austeridad fiscal permitieron recuperar parcialmente la estabilidad. En 1985, se firmó un acuerdo con el FMI y se implementaron un conjunto de reformas, incluyendo la liberalización del tipo de cambio. La combinación de políticas fiscales y monetarias, además de una reforma tributaria, incrementaron los ingresos tributarios, con efectos menos notorios sobre el crecimiento del PIB.

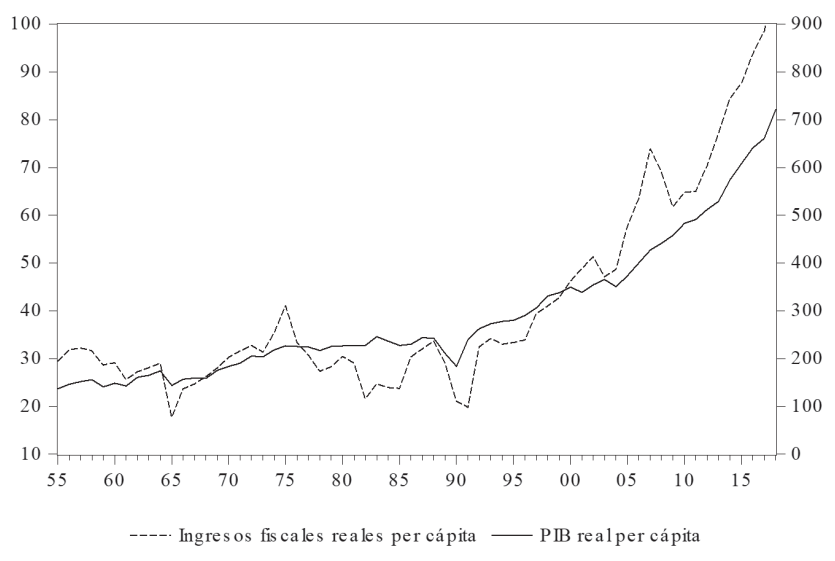

Figura 1. PIB real per cápita (RD\$, eje izquierdo) e ingresos fiscales para la RD (RD \$, $1990=100$ eje derecho)

Fuente: Fondo Monetario Internacional - Estadísticas Financieras Internacionales.

En 1990, el país experimentó otro colapso macroeconómico, esta vez debido a la crisis petrolera internacional que resultó cuando Irak invadiera Kuwait en agosto de ese año. Los niveles de inflación desde finales de la década de 1980 y el aumento en los 
precios del petróleo, causaron una grave crisis energética, lo que resultó en la mayor caída del PIB que haya vivido el país. Consecuentemente, los ingresos fiscales se desplomaron, hasta que en 1992 se firmó un nuevo acuerdo con el FMI y se implementó una ambiciosa reforma fiscal. Hubo un recorte sustancial en las tasas impositivas con una ampliación de la base fiscalizable que permitió aumentar sustancialmente los ingresos tributarios, mejorar la confianza y promover la inversión privada. Estas medidas pavimentaron el camino para una década de desarrollo y prosperidad que continuó hasta la crisis financiera de 2003-2004.

En 2002, debido a débiles regulaciones en el sector financiero, varios bancos que tenían operaciones paralelas en el extranjero, siguiendo un esquema de Ponzi, colapsaron a finales de ese ańo. El colapso bancario y varios intentos de estabilización fallidos con el FMI, provocaron una gran devaluación de la moneda que superó más del $60 \%$ entre 2002-2004. Curiosamente, ni la producción ni los ingresos tributarios cayeron en la magnitud de la crisis, aparentemente por el efecto que la devaluación tuvo sobre la cuenta corriente, los impuestos que provenían de las importaciones de petróleo, y la dinámica de otros sectores transables que se beneficiaron de la devaluación de la moneda.

Finalmente, los ingresos tributarios se vieron dramáticamente afectados por la crisis financiera mundial de 2008, como resultado de la caída del turismo y las remesas internacionales. Sin embargo, el PIB no se vio afectado gracias a una política fiscal expansiva que inicio en 2009, siguiendo las recomendaciones de varios organismos internacionales, con el objetivo de mitigar el posible desbordamiento de la situación internacional sobre la economía dominicana.

Por otro lado, la figura 2 muestra la presión tributaria y la inflación para el período de análisis. Esta comparación proporciona una perspectiva diferente sobre el éxito de las reformas tributarias con relación a la evolución de los ingresos. La tasa impositiva promedio, medida por la presión tributaria, se encontraba en su nivel más alto durante el régimen dictatorial de Trujillo. Las recaudaciones de impuestos disminuyen significativamente en comparación con el PIB durante la crisis política, alcanzando su punto más bajo en 1965 al comienzo de la guerra civil.

Los ingresos fiscales se restablecieron después de 1965, cuando se introduce la nueva constitución y un nuevo código tributario. Después de alcanzar su máximo post dictadura en 1975, la presión tributaria comenzó a disminuir debido a la caída de los ingresos fiscales en un entorno caracterizado por una fuerte devaluación de la moneda y altos niveles de inflación (el "efecto" Oliviera-Tanzi).

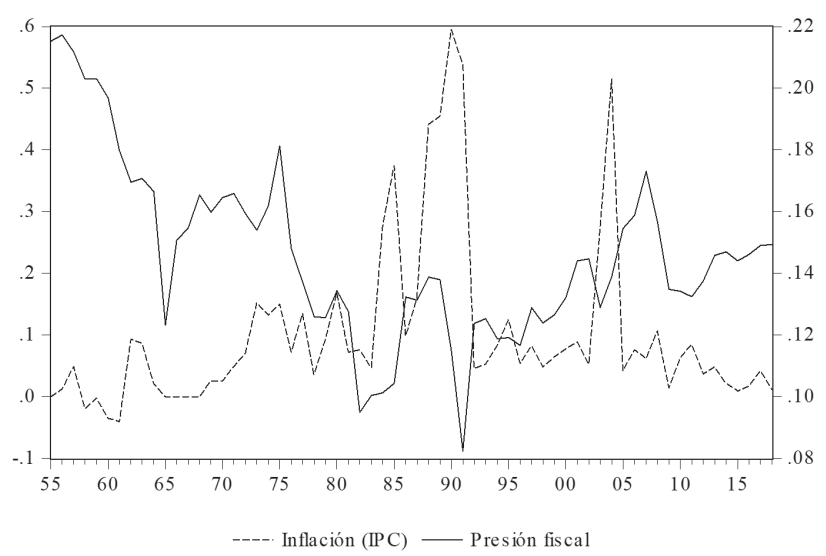

Figura 2. Presión tributaria (\%, eje izquierdo) e inflación del IPC (cambios \%, eje derecho) para la RD.

Fuente: Fondo Monetario Internacional - Estadísticas Financieras Internacionales.

La presión fiscal continuó cayendo hasta alcanzar otro mínimo en 1982 al comienzo de la crisis de esa década. Aparentemente, el impuesto inflacionario generado por la crisis permitió que los ingresos fiscales superaran la caída del PIB. Sin embargo, en la crisis de 1990 los ingresos fiscales cayeron más que el PIB. La escasez de energía y otros factores de producción interrumpieron la absorción doméstica, causando una dramática caída en las recaudaciones. Por el contrario, la caída del PIB se vio atenuada 
en parte por el sector transable, lo que produjo una caída menos dramática que la observada en los ingresos fiscales.

Después de 1992, producto de la exitosa reforma tributaria y una inflación estable, la presión tributaria inicio un aumento sostenido que, sin tomar en cuenta la crisis financiera de 2003-2004, continuó hasta 2007. En 2008, se evidenció una ruptura en la tendencia, probablemente debido al estímulo económico resultante de una política fiscal expansiva. El aumento de la producción provino del estímulo del gobierno, que aparentemente produjo menos ingresos fiscales que si la misma proviniera del sector privado (un efecto desplazamiento o "crowding-out").

Finalmente, la figura 3 muestra la relación entre los ingresos y la presión tributarios. Esta gráfica cuenta una historia diferente sobre el entorno fiscal en la República Dominicana. La presión fiscal estuvo en su nivel más alto, relativo a los ingresos tributarios, durante la década los cincuenta, los que es consistente con una economía dictatorial, con un sector formal pequeño, en donde la mayor parte de la producción se realizaba por un número limitado de empresas controladas por el régimen.

Después de la caída del régimen dictatorial en 1962, la informalidad disminuyó gradualmente, a medida que la democracia se estableció y surgió un nuevo sector privado. El dinamismo de la inversión privada permitió diversificar la producción, haciendo que el PIB creciera más rápido que los ingresos fiscales. En consecuencia, la presión tributaria se redujo, mientras que los ingresos tributarios fluctuaron alrededor de su media.

Después de las reformas fiscales de 1992, con un sector privado más sólido y exportaciones provenientes de sector de servicios, tanto los ingresos fiscales como la presión tributaria comenzaron a moverse proporcionalmente. En 2005, sin embargo, se observa una ruptura estructural, y el PIB aparenta haber aumentado a un ritmo mayor que los ingresos fiscales, causando una caída en la presión tributaria.

Una posible interpretación de este comportamiento es que los sectores que contribuyeron a la expansión del PIB después de 2005 no lo hicieron proporcionalmente con sus compromisos tributarios. El aumento en los ingresos tributarios puedo ser el resultado de una sobrecarga de los sectores que ya pagaban impuestos, aun cuando la expansión del PIB provino de sectores donde predomina la evasión, elusión e informalidad, o que se benefician de subsidios tributarios.

De hecho, algunas estadísticas del Banco Central y el Ministerio de Hacienda de la República Dominicana mostraron que, en 2016, más del $60 \%$ de los sectores que contribuían al PIB tenían una presión tributaria inferior al $10 \%$, donde el $14.6 \%$ fue el promedio la presión tributaria en ese año. El $40 \%$ restante, sin embargo, eran sectores que tenían una presión tributaria que se extiende más allá del $30 \%$, incluidas las telecomunicaciones con una presión superior al $70 \%$, la manufactura con más del $55 \%$ y el sector bancario con alrededor del $45 \%$.

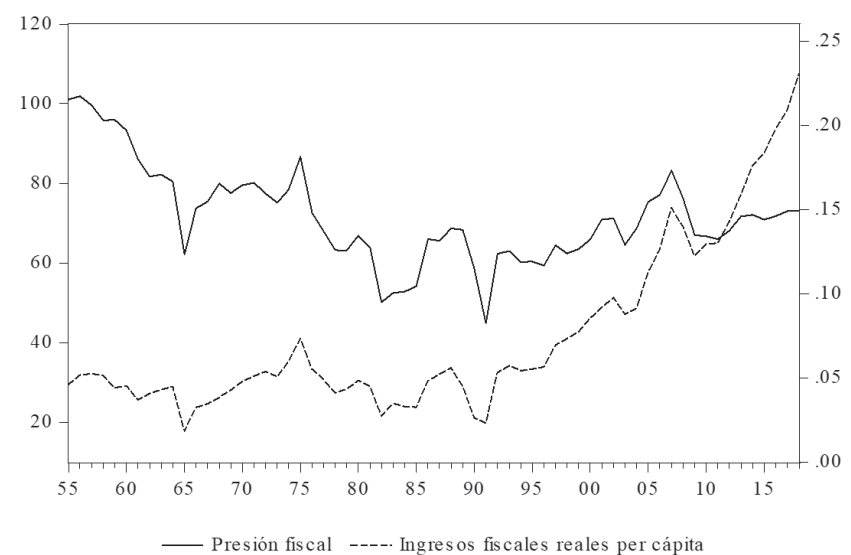

Figura 3. Presión fiscal (\%, eje izquierdo) e ingresos tributarios reales per cápita $(\mathrm{RD} \$, 2010=100$, eje derecho)

Fuente: Fondo Monetario Internacional - Estadísticas Financieras Internacionales. 
Esto sugiere que existen fuertes distorsiones en el sistema tributario dominicano, las cuales se pueden manifestar en la evasión y una economía informal significativa. Finalmente, la migración desde nuestro vecino, la República de Haití, con más de 2 millones de haitianos o el $20 \%$ de la población total del país, se encuentra en el centro del problema de la informalidad que enfrenta la economía dominicana.

\section{La curva de Laffer}

La figura 4 muestra una representación de la curva de Laffer (1981). Los extremos, con niveles de impuesto de $0 \%$ y $100 \%$, están asociados con cero ingresos fiscales. Esto se desprende de la lógica de que, sin impuestos, el gobierno no recaudaría ingresos tributarios. ${ }^{5}$ Además, si el gobierno apunta a una presión tributaria del $100 \%$, no habrá incentivos para la producción ni el trabajo, lo que resultaría también en un escenario de completa asfixia fiscal, sin recaudaciones tributarias. Entre estos dos extremos, sin embargo, se supone que debe existir una presión tributaria que maximiza los ingresos fiscales del gobierno.

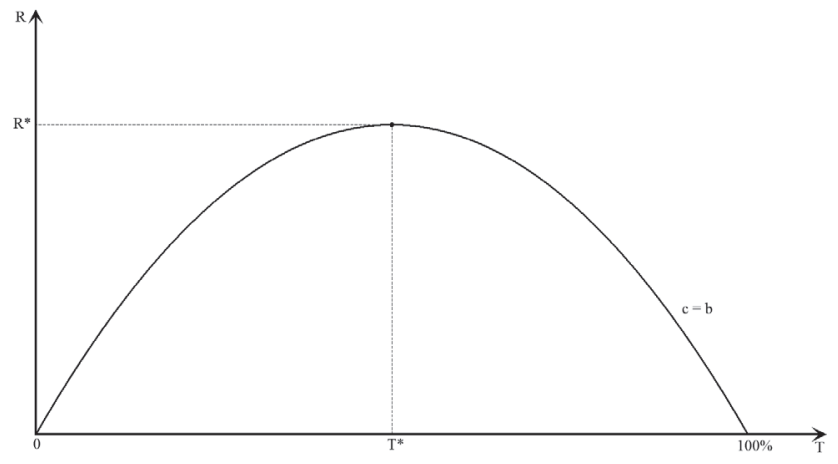

Figura 4. La curva de Laffer

Fuente: Laffer (2004).

5. En algunos modelos teóricos, la curva de Laffer puede inclinarse hacia arriba hasta una tasa impositiva del $100 \%$ (Malcomson, 1986). Wanniski (1978) también observó que era poco probable que la actividad económica cesara al 100 \% de los impuestos, cambiando del intercambio de dinero a una economía de trueque. También puede haber circunstancias especiales, como los tiempos de guerra, en los que la actividad económica puede continuar con una tributación cercana al $100 \%$.
La curva de Laffer se puede representar y es compatible con una familia de funciones matemáticas llamadas polinomios uni-variables. Dentro de esta clase de polinomios, la función cuadrática es la representación más obvia para la curva de Laffer. ${ }^{6}$

$$
R=b T-c T^{2}
$$

La ecuación (1) es una función cuadrática, donde $R$ representa los ingresos fiscales del gobierno y $T$ la presión tributaria. ${ }^{7}$ Los parámetros $b$ y $c$ dan la forma que caracteriza la curva, y muestran la interacción entre el efecto aritmético y el efecto económico de los impuestos.

El efecto aritmético, representado por el término $b T$, mide el impacto lineal de variar los impuestos sobre los ingresos fiscales. $\mathrm{Si}$, por ejemplo, un aumento en los impuestos hace que la presión tributaria aumente en un $1 \%$, los ingresos fiscales aumentarán linealmente en $b \times(1 \%)$. El efecto económico, sin embargo, supone que los impuestos afectarán el comportamiento de los contribuyentes de forma no lineal. A medida que los impuestos se elevan, se deprime el incentivo de los empleados para trabajar y el de las empresas para producir. Por su naturaleza no lineal, llegará un punto donde el efecto económico será mayor que el efecto aritmético, provocando que los ingresos fiscales eventualmente disminuyan. Esto implica que el efecto económico es negativo y exponencial, de manera que $-c T^{2}$. Combinando el efecto económico con el efecto aritmético, se produce un vértice que da la forma parabólica a la curva de Laffer. Según este vértice, la

6. La figura 4 muestra la representación que tradicionalmente aparece en los libros de texto, en donde $c=b$, produciendo un impuesto óptimo al nivel de $50 \%$.

7. Aunque en el contexto tradicional de la curva de Laffer, $T$ representa la tasa impositiva, en este contexto, la presión tributaria se utiliza como un proxy de la tasa impositiva promedio de la economía. La presión tributaria se define como el cociente entre los ingresos tributarios y el Producto Interno Bruto (PIB). 
presión tributaria que maximiza las recaudaciones fiscales está dada por:

$$
T^{*}=\frac{b}{2 c}
$$

En adición a la presión tributaria que maximiza los ingresos fiscales, la ecuación (1) se puede factorizar para encontrar las presiones tributarias en las que los ingresos fiscales son cero. La factorización produce dos soluciones. La primera, se ubica en el origen cuando $T^{o}=0$. La segunda, que es el punto de intercepción ubicado a la derecha del vértice, viene dada por:

$$
T^{u}=\frac{b}{c}
$$

El punto de intercepción superior, dado por la ecuación (3), es relevante ya que la curva de Laffer puede tener un efecto económico dominante que ocasiona que los ingresos fiscales caigan de manera precipitada. Un efecto económico dominante, puede ser el resultado de diferentes factores, incluyendo distorsiones fiscales en la forma de evasión, elusión e informalidad (Feige y McGee, 1982a, 1982b y 1983).

Si existen distorsiones fiscales en una economía, el aumento de los impuestos podría ocasionar que los ingresos tributarios comiencen a disminuir antes de lo esperado, alcanzando un máximo que será menor que el que se obtendrían en una economía con menos distorsión (Gutmann, 1977 y Fullerton, 2008). En este sentido, los cambios en el efecto económico pueden proporcionar información útil sobre la magnitud de las distorsiones en una economía.

Por ejemplo, la figura 5 compara una curva de Laffer en la que el efecto aritmético y económico son iguales $(c=b)$ con una en la que el efecto económico domina $(c>b) .{ }^{8}$ Evidentemente, una $c$ mayor reduce la presión tributaria que maximiza las recaudaciones, del nivel $T^{*}$ al nivel $T^{*}$, y el ingreso fiscal máximo del punto $A$ al punto $B .^{9}$ Un efecto económico dominante, puede ser interpretado como una situación en la que la estructura de la economía no puede manejar los impuestos, ni entregar el mismo nivel de ingresos fiscales que en países con entornos fiscales menos distorsionados. ${ }^{10}$

Para introducir formalmente las distorsiones fiscales en el modelo, se asume que existe una curva de Laffer objetiva, pero "no observada", dada por:

$$
\tilde{R}=b \tilde{T}-c \tilde{T}^{2}
$$

donde $\tilde{R}$ y $\widetilde{T}$ son los niveles "no observados" de ingresos fiscales y presión tributaria que se obtendrían en la ausencia de distorsiones fiscales. También se asume que los ingresos fiscales "observados" están dados por:

$$
R=\widetilde{R}(1-\varepsilon)
$$

8. Cuando $c=2 b$, la intercepción superior es 1 . En la figura 5 , se supone, que $c=2 b$.

9. Este resultado es consistente con Friedman et al., (2000), en el que la evasión fiscal está presente en países con legislaciones deficientes y gobiernos corruptos, lo que hace que la curva de Laffer se encoja como lo muestra la figura 5.

10. Este argumento es factible ya que los países en vías de desarrollo sufren distorsiones sustanciales en sus sistemas tributarios, y tienen, en promedio, presiones fiscales mucho más bajas que los países desarrollados, con instituciones y sistemas judiciales más sólidos. Por ejemplo, la presión fiscal en América Latina fue en promedio del $23 \%$ para el período entre 2000 y 2015 según las estadísticas financieras internacionales del FMI, mientras que la presión fiscal para los países de la OCDE fue mucho más del $36 \%$ en el mismo período. Los estudios empíricos sobre la curva de Laffer muestran que la presión fiscal óptima en algunos países desarrollados puede llegar al $70 \%$ como Suecia (Stuart, 1981) y alrededor del $65 \%$ en países con una sólida acumulación de capital humano (Pecorino, 1995). 
donde $0 \leq \varepsilon \leq 1$ es el nivel de distorsiones fiscales en la economía. De esto se deduce que la presión tributaria está dada por: $\tilde{T}=\tilde{R} / Y$, donde $Y$ es el producto interno bruto (PIB) de la economía. ${ }^{11}$ Sustituyendo $\tilde{R}$ y $\tilde{T}$, que están dados por $\tilde{R}=R /(1-\varepsilon)$ y $\tilde{T}=T /(1-\varepsilon)$, en la ecuación (3) y simplificando, se obtiene que:

$$
R=b T-\frac{c}{(1-\varepsilon)} T^{2}
$$

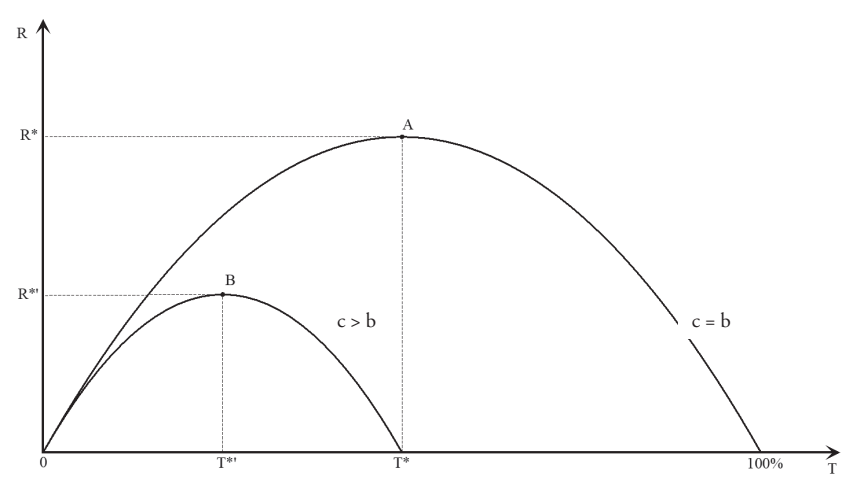

Figura 5. distorsiones fiscales y la curva de Laffer Fuente: Laffer (2004) y diagrama del autor.

La ecuación (6) se puede reducir a una función "observable" dada por:

$$
R=\hat{b} T-\hat{c} T^{2}
$$

donde $\hat{b}=b$ y $\hat{C}=c /(1-\varepsilon)$. La ecuación (7) muestra que, en la presencia de distorsiones fiscales, con $\varepsilon>0$, el efecto económico observado $(\hat{c})$ aumentará

11. Hay que tener en cuenta que se ha hecho es supuesto de que las distorsiones fiscales afectan los ingresos fiscales y no el PIB. La lógica detrás de este argumento es que aquellos contribuyentes que evaden impuestos realmente generan la producción que creó el impuesto. En adición, se podría afirmar que, si los impuestos son utilizados de manera ineficiente por el gobierno, la evasión fiscal podría aumentar la producción, ya que podría ser utilizada de manera más eficiente por el evasor de impuestos. Sin embargo, en las economías donde el gobierno es eficiente en la distribución de los ingresos fiscales a través de inversiones productivas, las distorsiones fiscales podrían tener un efecto negativo sobre el PIB. $y$, por consiguiente, tanto la presión tributaria que maximiza los ingresos, así como los ingresos fiscales máximos, caerán. De esto se deduce que, la presión tributaria óptima, en la presencia de distorsiones fiscales, está dada por:

$$
\widehat{T}^{*}=\frac{\hat{b}}{2 \hat{c}} \equiv \frac{b(1-\varepsilon)}{2 c}
$$

Si la economía presenta una curva de Laffer con sus correspondientes efectos aritmético y económico, se puede estimar la ecuación (7) y calcular la presión tributaria óptima. Además, si existen distorsiones fiscales en la economía, las ecuaciones (3) y (7) pueden utilizarse para aproximar el grado de tales distorsiones.

Si bien el verdadero nivel de distorsiones fiscales será difícil de estimar, la ecuación (7) puede usarse para medir el nivel de distorsiones que, de eliminarse, resultaría en una curva de Laffer que tiene su extremo superior en el $100 \%$. Esto se logra factorizando la ecuación (6), estableciendo el límite superior a la unidad, de manera que $T^{u}=1$, y aislando el valor de $\boldsymbol{\varepsilon}$.

Los resultados de esta manipulación darían el nivel de distorsiones fiscales que hace que la curva de Laffer, en la que $c=b$, se reduzca a la curva de Laffer observada con $\hat{c}>\hat{b}=b$ y con $\hat{c}=c /(1-\varepsilon)$. Después de factorizar la ecuación (6), se tiene que:

$$
\varepsilon=1-\frac{\hat{c}}{\hat{b}}
$$

donde $\hat{c}$ y $\hat{b}$ son los parámetros empíricos luego de estimar la ecuación (7). La ecuación (9) es una aproximación de las distorsiones fiscales en la economía, las cuales, según los argumentos presentados, reducen la capacidad del gobierno para presionar por impuestos más altos y recaudar mayores ingresos 
fiscales. Aunque la ecuación (9) probablemente no mida el verdadero nivel de distorsiones fiscales en la economía, las misma podría usarse para estudiar la evolución temporal de la aproximación de dichas distorsiones, y su relación con el desempeńo de la economía, con choques exógenos y con reformas macroeconómicas.

\section{La curva de Laffer en la República Dominicana}

Esta sección generaliza el enfoque de Hsing (1996) para estimar una curva de Laffer para la República Dominicana. Utilizando la especificación de Bårdsen (1989), se empleó una versión cointegrada de la ecuación (7):

$$
\begin{aligned}
\Delta R_{t}= & \sum_{i=0}^{n}\left(\widehat{\delta}_{1 i} \Delta R_{t-i-1}+\widehat{\delta}_{2 i} \Delta T_{t-i-1}+\widehat{\delta}_{3 i} \Delta T_{t-i-1}^{2}\right) \\
& -\hat{\phi}\left(R_{t-n}-\hat{\beta} T_{t-n}+\hat{\gamma} T_{t-n}^{2}\right)
\end{aligned}
$$

donde $R_{t}$ son los ingresos tributarios per cápita en términos reales, $T_{t}$ es la presión tributaria, medida como la razón de $R_{t}$ sobre el PIB, y $\Delta$ es un operador de primeras diferencias. ${ }^{12}$ La curva de Laffer de largo plazo estaría dada por:

$$
R_{t}=\hat{b} T_{t}-\hat{c} T_{t}^{2}
$$

que resulta cuando todas las variaciones en (10) tienden a cero. La ecuación (11) es análoga con la ecuación (7), donde $\hat{b}=\hat{\beta} /-\hat{\phi}$ y $\hat{c}=\hat{\gamma} /-\hat{\phi}$.

La ecuación (10) se estimó utilizando datos anuales entre 1955 y 2018. El primer paso en el proceso de estimación es probar la coherencia en la integración

12. La estimación de la ecuación (10) incorporará una constante y supone que los errores son independientes e idénticamente distribuidos. Para poder comparar la magnitud de los coeficientes, los ingresos fiscales reales per cápita se escalan a 1 en 1000. Los datos provienen de las Estadísticas Financieras Internacionales del FMI. de las series. La tabla 1 muestra una prueba aumentada de Dickey y Fuller (1979). Los resultados indican que todas las variables son integradas de primer orden $I(1)$, lo que proporciona un conjunto coherente de procesos estacionarios en primeras diferencias, que es una condición necesaria para la integración del modelo.

Tabla 1. Prueba ADF en variables seleccionadas

\begin{tabular}{cc}
\hline Variable & ADF \\
\hline$R_{t}$ & -2.20 \\
$\Delta R_{t}$ & $-6.39^{*}$ \\
$T_{t}$ & -2.85 \\
$\Delta T_{t}$ & $-8.34^{*}$ \\
& \\
$T_{t}^{2}$ & -2.97 \\
$\Delta T_{t}^{2}$ & $-8.07^{*}$
\end{tabular}

Fuente: cálculos del autor.

Notas: * denota significancia al $5 \%$ bajo los valores críticos de MacKinnon (1996). La prueba incluye una constante, sin tendencia y con un máximo de 9 rezagos seleccionado usando el Criterio de Información de Schwarz (SIC).

Por otro lado, la ecuación (12) presenta la estimación del modelo utilizando mínimos cuadrados en dos etapas. Además, se incluyó una tendencia para capturar los posibles cambios tecnológicos y de productividad en el sistema tributario.

$$
\begin{gathered}
\Delta R_{t}=-\underbrace{0.05}_{(0.02)}-\underbrace{0.21}_{(0.08)} R_{t-1}+\underbrace{0.51}_{(0.21)} T_{t-1}-\underbrace{1.28}_{(0.69)} T_{t-1}^{2}+\underbrace{0.33}_{(0.09)} t \\
N=61 A R^{2}=0.35 \sigma=3.85 \\
F=8.85(p<0.001) \text { Rank }=14 J=34.97(p<0.001)
\end{gathered}
$$

Todas las variables son significativas más allá de un nivel crítico del $1 \%$ y tienen los signos esperados. La ecuación captura aproximadamente el $35 \%$ de las variaciones en los ingresos tributarios reales per cápita que, para un modelo en primeras diferencias, 
proporciona un ajuste adecuado. Todas las estadísticas están en orden, particularmente los indicadores Fy $J$.

El coeficiente del ingreso fiscal per cápita real rezagado, $\phi$, es negativo y se encuentra dentro del círculo unitario, lo que indica que la ecuación de cointegración es estable. Su magnitud implica que aproximadamente el $21 \%$ de cualquier desviación respecto al nivel de equilibrio, se corrige antes del primer año.

Después de encontrar la ecuación cointegrada para la curva de Laffer, se realizó una prueba aumentada de Dickey y Fuller (1979) sobre los residuos de la ecuación (12). Los resultados confirman residuos estacionarios con $t=-4.99$ y $\mathrm{p}<0.01$ según la prueba de MacKinnon (1996). Engle y Granger (1987) muestran que este resultado proporciona una condición necesaria para la cointegración.

Tabla 2. Prueba de cointegración

\begin{tabular}{cccccc}
\hline \multirow{2}{*}{$\begin{array}{c}\text { Hipótesis } \\
\text { No. of } \\
\text { ECM }\end{array}$} & Eigen- & \multicolumn{2}{c}{ Trazo } & \multicolumn{2}{c}{ Max Eigen-valores } \\
\cline { 3 - 6 } & valores & Estadística & $\begin{array}{c}5 \% \\
\text { Valor } \\
\text { Crítico }\end{array}$ & Estadística & $\begin{array}{c}\text { Valor } \\
\text { Crítico }\end{array}$ \\
\hline Ninguno & 0.33 & $47.77^{*}$ & 29.80 & $24.67^{*}$ & 21.13 \\
Máximo 1 & 0.24 & $23.10^{*}$ & 15.50 & $16.36^{*}$ & 14.27 \\
Máximo 2 & 0.11 & $6.74^{*}$ & 3.84 & $6.74^{*}$ & 3.84 \\
\hline
\end{tabular}

Fuente: cálculos del autor.

Notas: Max Eigen-valores y el trazo indican un vector de cointegración al $5 \%$. ${ }^{*}$ denota el rechazo de la hipótesis de no cointegración a $5 \%$ usando la prueba de MacKinnon Haug y Michelis, (1999).

La tabla (2) presenta la prueba de Johansen (1991), que confirma la existencia de por lo menos una relación cointegrada. La ecuación normalizada obtenida de (12) está dada por:

$$
R_{t}=\underbrace{2.45}_{(0.99)} T_{t}-\underbrace{6.13}_{(-2.96)} T_{t}^{2}
$$

La ecuación (13) muestra el efecto aritmético y el efecto económico de la tributación de largo plazo, que están dados por $\hat{b}=2.45$ y $\hat{c}=6.13$, respectivamente. El coeficiente de la presión tributaria es positivo y significativo, evidenciando el efecto aritmético de la tributación. De igual manera, el coeficiente del cuadrado de la presión tributaria es negativo y significativo, lo que indica que existe un efecto económico en el sistema tributario dominicano. ${ }^{13}$

La magnitud del efecto económico es casi tres dos veces y media mayor que el efecto aritmético, lo que implica que los esfuerzos para aumentar la presión tributaria, bajo las características estructurales de la economía dominicana, serán mermados, en gran medida, por el incentivo de los contribuyentes y las empresas para evadir impuestos e informalizarsce.

Usando los coeficientes de la ecuación (13) en la ecuación (2), la presión tributaria que maximizaría los ingresos fiscales del gobierno se estima en $20 \%$. La magnitud del impuesto de Laffer aparenta ser consistente con las limitaciones estructurales que presenta la economía dominicana, dado que la presión tributaria real fue de aproximadamente un $15 \%$ en 2018 y no ha podido superar un nivel mayor al $18 \%$ en la era posterior a la dictadura.

Además de estimar el impuesto de Laffer, el modelo permite medir la presión tributaria máxima que causaría un desplazamiento total de los ingresos fiscales.

13. Los errores estándar de los coeficientes normalizados en la ecuación (13) provienen de la fórmula de Bårdsen (1989) para la varianza. Como ejemplo, la variación del efecto económico a largo plazo está dada por:

$$
\begin{array}{r}
\operatorname{var}(\hat{b}) \cong\left(\frac{1}{\hat{\phi}}\right)^{2} \operatorname{var}(\hat{b})+\left(\frac{\hat{b}}{\hat{\phi}}\right)^{2} \operatorname{var}(\hat{\phi}) \\
+2\left(\frac{1}{\hat{\phi}}\right)\left(\frac{\hat{b}}{\bar{\phi}}\right) \operatorname{cov}(\hat{b}, \hat{\phi})
\end{array}
$$

donde los parámetros y la matriz de covarianza se obtienen de la estimación de la ecuación (12). 
Usando la ecuación (3) y los parámetros obtenidos de la ecuación (13), la presión fiscal máxima, a la cual la curva de Laffer cae a cero, se estima en $40 \%$. Este nivel es bajo en comparación con economías industrializadas y es indicativo de la gran sensibilidad del sistema tributario al efecto económico de la tributación, lo que sugiere la existencia de distorsiones significativas.

Las distorsiones fiscales pueden provenir de diferentes fuentes, con la evasión, elusión e informalidad como las más evidentes y relevantes para este ejercicio. Al igual que muchos países en vía de desarrollo, la República Dominicana parece sufrir de distorsiones fiscales (Prazmowski, 2014). En 2018, la República Dominicana ocupó el lugar 129 entre 180 países en la lista de los más corruptos. Además, en una escala de 100 , el país obtuvo un puntaje de 30 , donde 100 representan el nivel más alto de transparencia en la conducción de las políticas gubernamentales (Transparency International, 2018). Ciertamente, estas distorsiones debilitan la efectividad de la política fiscal e incentivan a los contribuyentes a justificar formas de evadir impuestos (Gërxhani y Schram, 2006; Torgler, 2005). Si este es el caso, el modelo sugiere que las distorsiones fiscales producirían un efecto económico mayor al efecto aritmético sobre los ingresos fiscales.

Usando la ecuación (9) y los parámetros estimados del modelo, la magnitud de las distorsiones fiscales puede ser aproximada. Al 2018, se estima que la evasión, elusión y la informalidad combinadas se encuentran en un rango de entre $46 \%$ y $70 \%$ con una media de $60 \%$, representando el porcentaje de la actividad económica que las autoridades tributarias no están o no pueden fiscalizar. ${ }^{14}$ Este

14. Estas cifras son similares a las obtenidas en otros estudios como es el de Waseem (2018) para el caso de Pakistán o para las estimaciones que resumen Fuest \& Riedel (2009) en un recuento de la literatura sobre evasión, elusión e informalidad para países en vía de desarrollo. elevado nivel de distorsiones, que aun en su rango inferior es alto, es quizás la razón por la cual el efecto económico de los impuestos es tan grande y la presión tributaria ha sido históricamente tan baja. En este sentido, la evasión, elusión e informalidad representan entre un $9 \%$ y un $25 \%$ del PIB con un rango promedio de un $15 \%$. Por lo tanto, si no hubiesen distorsiones, el fisco pudiese duplicar sus recaudaciones o, alternativamente, podría recaudar el mismo nivel de ingresos tributarias reduciendo a la mitad, ceteris paribus, las tasas impositivas.

Más interesante, sin embargo, es la evolución histórica de las distorsiones. Usando técnicas recursivas, se estimaron los coeficientes de las ecuaciones (12) y (13), y se calcularon las distorsiones fiscales a lo largo del período de estimación. La figura (6) muestra la evolución de estas distorsiones desde 1968 hasta 2018 junto con sus respectivas bandas de error.

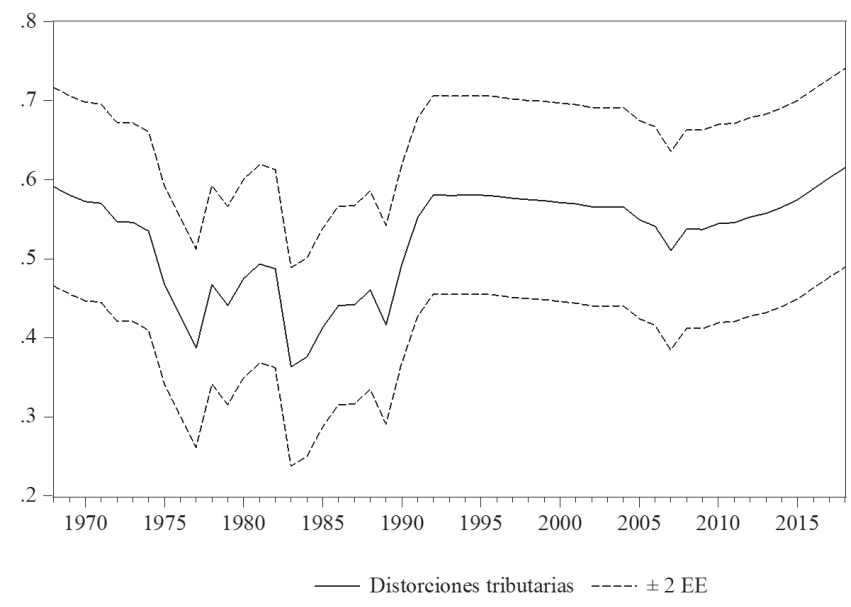

Figura 6. Estimación de coeficientes recursivos de distorsiones tributarias en la RD.

Fuente: cálculos del autor.

El gráfico proporciona una historia muy convincente que se relaciona estrechamente con la historia del sistema tributario dominicano. Al comienzo de la muestra, en 1968, las distorsiones fiscales eran de un orden similar $(60 \%)$ al actual, y empezaron 
a disminuir gradualmente a medida que la democracia y un nuevo sector empresarial comenzaron a establecerse. $^{15}$

Varias reformas fiscales y modificaciones al código tributario entre 1970 y 1975 permitieron mejorar la recaudación de impuestos e incentivar la inclusión de contribuyentes potenciales al sistema. Sin embargo, después de 1975, hubo un resurgimiento de las distorsiones, que aparentemente reaccionó a cambios importantes en el sistema tributario, incluyendo un aumento en los impuestos al consumo, los impuestos ad valoren, los impuestos a los combustibles y las restricciones de precios destinadas a aumentar los ingresos fiscales del gobierno. Además, hubo una disminución sustancial, debido a la caí$\mathrm{da}$ en los precios internacionales de los productos básicos, en la rentabilidad de los sectores agrícola y de exportación. El incremento de los impuestos y el deterioro de las condiciones económicas, probablemente llevaron muchos sectores a la informalidad, impulsando el efecto económico de los impuestos, e incentivando a los contribuyentes a evadir sus compromisos tributarios.

En 1983, se implementó una reforma tributaria importante que introdujo un impuesto a las transferencias de bienes y servicios (ITBIS), un aumento sustancial en el impuesto sobre la renta, impuestos específicos a los cigarrillos y el alcohol, y una modificación a la tasa cambio oficial que se usaba como referencia para calcular los impuestos a las importaciones. Este choque tributario ciertamente contribuyó a un aumento gradual en la evasión y la informalidad.

Durante 1985 y hasta 1990, la economía dominicana experimentó una importante crisis econó-

15. El gráfico comienza en 1968 porque se pierden 13 observaciones como grados de libertad para obtener la primera estimación recursiva. mica. La situación requirió reformas sustanciales, impuestas por un acuerdo con el FMI, que comenzó en 1991. El paquete de estabilización requirió cambios fiscales y monetarios para recuperar la estabilidad macroeconómica, además de una reforma salarial para compensar la pérdida del poder adquisitivo producida por la crisis.

Una de las transformaciones más significativas fue en el sistema tributario. Las tasas de impuestos, tanto en el ITBIS como en el impuesto sobre la renta, se redujeron y la base imponible aumentó. Estas modificaciones, así como una simplificación sustancial del código tributario, ayudaron a regular y mejorar los ingresos tributarios, lo que permitió al gobierno alcanzar un presupuesto equilibrado. Las medidas se reflejaron en las distorsiones fiscales, que comenzaron a caer a medida que la confianza en las nuevas políticas se fue consolidando.

La caída en las distorsiones fiscales continuó y se acentuó a partir del 2004, cuando una nueva tecnología, basada en el uso de comprobantes fiscales, permitió mejorar los ingresos tributarios al tiempo que reducía la evasión, elusión e informalidad. Los efectos en la recaudación de impuestos fueron notables, sin embargo, los nuevos controles pronto se relajaron después de que se eligiera un nuevo gobierno en 2006.

En respuesta a la crisis financiera internacional de 2007-2008, el gobierno dominicano inició un agresivo plan de política fiscal expansiva, que resultó en importantes déficits financiados con deuda. Esta política ha continuado y en 2016 un escándalo mayor con el contratista brasileño Odebrecht reveló la existencia de un serio problema de corrupción y la malversación de fondos (Transparency international, 2016).. Se puede argumentar que esta situación, que parece haber comenzado en 2008, pudo contribuir con el aumento notorio que se observó en las distorsiones fiscales. 


\section{Distorsiones fiscales y sus implicaciones para el diseño de políticas}

Claramente, las distorsiones fiscales erosionan significativamente los ingresos fiscales y la capacidad de los gobiernos para funcionar e implementar políticas sociales. Esta sección describe el efecto de las distorsiones fiscales sobre la curva de Laffer y sus consecuencias en el costo de oportunidad que incurren los gobiernos para recaudar impuestos. La figura 7 compara la curva de Laffer bajo la estructura tributaria actual, con aquella que resultaría de una reducción en las distorsiones fiscales.

Evidentemente, el nivel de distorsiones fiscales no solo aumenta el efecto económico de los impuestos, sino que reduce el potencial de ingresos fiscales para los gobiernos. A medida que se reducen las distorsiones fiscales, los ingresos fiscales aumentan y la curva de Laffer se desplaza hacia la derecha. Sin embargo, debido a que la presión tributaria es endógena en este modelo, ya que $T=R / \bar{Y}$, el análisis proporciona un sistema simultáneo que crea un efecto multiplicador de las políticas dirigidas a reducir las distorsiones fiscales.

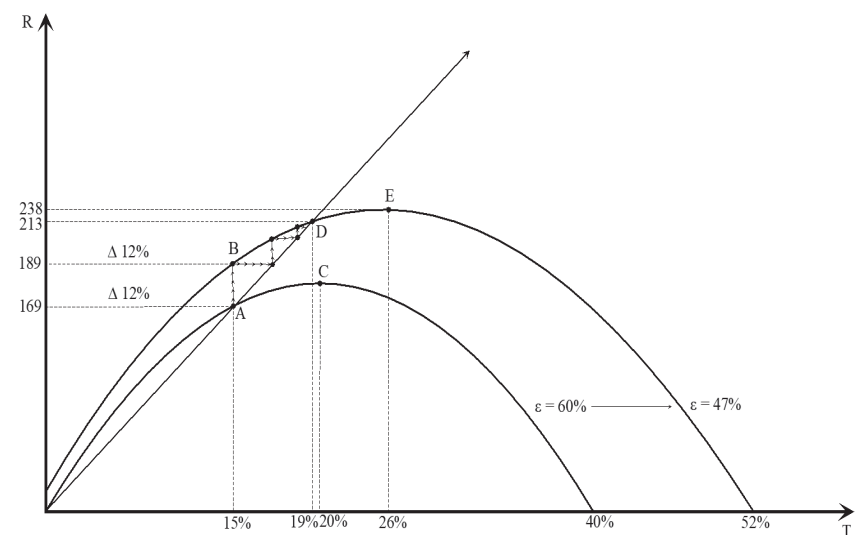

Figura 7. La curva de Laffer y los cambios en las distorsiones fiscales.

Fuente: Laffer (2004) y diagrama del autor.

El efecto de cambiar los niveles de las distorsiones en el nivel resultante de los ingresos fiscales y la presión tributaria se obtiene al sustituir la ecuación (13) en $T=R / \bar{Y}$, mientras se factoriza el nivel de la presión tributaria. La factorización da dos soluciones. El primero está en el origen con $T^{\prime}=0$ y el segundo está dado por:

$$
T^{\prime \prime}=\frac{(\hat{b}-\bar{Y})(1-\varepsilon)}{\hat{b}},
$$

que en la figura 7 corresponde a los puntos (A) o (D) dependiendo del nivel de $\boldsymbol{\varepsilon}$. La ecuación (14) muestra que a medida que disminuyen las distorsiones fiscales, la presión fiscal, referente al nivel inicial del PIB dado por $\bar{Y}$, convergerá, ceteris paribus, a un nivel más alto dado por el punto D. Sin aumentar las tasas de impuestos, si se introducen políticas para abordar las distorsiones directamente, la relación entre los ingresos fiscales y la presión tributaria creará un efecto dinámico que impulsará tanto los ingresos fiscales como la presión tributaria de su nivel original $\mathrm{A}$, a un nuevo equilibrio en el punto D. La dinámica es estable y continuará hasta alcanzar el nuevo equilibrio.

El cambio inicial en la curva de Laffer aumentará los ingresos fiscales de A hacia B. Este mayor nivel de ingresos fiscales se refleja en la línea $T=R / \bar{Y}$, que luego produce un nuevo, y más elevado nivel de ingresos fiscales en la curva de Laffer modificada. En este sentido, el proceso continuará hasta que se logra la convergencia en el punto $\mathrm{D}$, destacando los beneficios de reducir las distorsiones fiscales en los ingresos y la presión tributaria.

Los gobiernos de países en vías de desarrollo, como la República Dominicana, se quejan de las bajas presiones tributarias y de la necesidad de mayores impuestos. Los nuevos impuestos, por lo general, recaen sobre sectores fácilmente fiscalizables, generando una gran desproporcionalidad, y el fomento hacia la evasión, elusión e informalidad. Las conclusiones de este análisis sugieren que una estrategia mucho más exitosa para aumentar la presión tributaria sería la de focalizar esfuerzos para reducir la evasión fiscal y fomentar la formalidad en los dife- 
rentes sectores de la economía (ver argumentos simulares en Busato y Chiarini, 2013).

Usando el modelo, se muestra que una reducción del $12 \%$ en las distorsiones fiscales, pasando de un $60 \%$ a $48 \%$, puede aumentar inicialmente los ingresos fiscales en $12 \%$, pasando del punto $\mathrm{A}$ al punto B. Sin embargo, el proceso de retroalimentación entre los ingresos fiscales y la presión tributaria permite alcanzar el equilibrio en el punto $\mathrm{D}$, lo que produce un aumento adicional del $12 \%$ en los ingresos tributarios. El efecto combinado es un aumento del $24 \%$, con una presión tributaria que va desde su nivel inicial de $15 \%$ a $19 \%$ sin la necesidad de aumentar las tasas de impuestos.

Hay que tener en cuenta que, al reducir las distorsiones fiscales, el incremento de los ingresos fiscales es casi el doble que el efecto de una política que intenta solo aumentar las tasas impositivas. Si el gobierno reduce las distorsiones fiscales, tendrá una mejor oportunidad de alcanzar una presión fiscal mayor a medida que se reduce el efecto económico de la tributación.

El mismo argumento sigue en la dirección opuesta. Si la economía estuviese una presión tributaria del $19 \%$ y los factores exógenos hacen que aumenten las distorsiones fiscales, el cambio en la curva de Laffer causará que la tasa impositiva y la presión tributaria caigan del punto $\mathrm{D}$ al punto $\mathrm{A}$, debido al proceso de convergencia de los ingresos fiscales sobre la presión tributaria.

Por lo tanto, las distorsiones fiscales no solo causan un costo de oportunidad directo para la economía, sino que, en el contexto de la presente investigación, producen un efecto multiplicador que va en la dirección opuesta a los cambios en las distorsiones fiscales. Si aumentan las distorsiones, el efecto multiplicador impulsará una disminución en los ingresos fiscales y una disminución en la presión tributaria. $\mathrm{Si}$, por el contrario, el gobierno logra reducir las distorsiones fiscales, tanto los ingresos fiscales como la presión tributaria aumentarán a medida que la curva de Laffer se desplace hacia la derecha.

Se puede argumentar que la razón por la cual el aumento de la presión tributaria en la República Dominicana ha sido desafiante, independientemente de muchas reformas fiscales, se debe a que los efectos multiplicadores de las distorsiones han sido ignorados. El análisis muestra que reducir las distorsiones fiscales podría ayudar a lograr mayores recaudaciones que aumentando impuestos, lo que a su vez permitiría dimensionar un Estado capaz de realizar inversiones en sectores relevantes para el desarrollo del país. ${ }^{16}$

\section{Conclusiones}

Se estimó una curva de Laffer para el sistema tributario dominicano y se determinó que la presión tributaria que maximiza los ingresos fiscales es de alrededor de un $20 \%$. También se encontró que el efecto económico de los impuestos es significativamente mayor que el efecto aritmético, lo que explica la anémica y extremadamente baja presión tributaria en el país. El modelo sugiere que la razón de un efecto económico dominante se debe a distorsiones fiscales que provienen de diferentes fuentes, incluyendo la evasión, elusión e informalidad.

Se estima que las distorsiones son del orden del $60 \%$. También se encontró que la evolución de las distorsiones fiscales corresponde con la evolución económica del país, incluyendo más de 20 reformas tributarias y 5 acuerdos de estabilización con el FMI. Después de caer por más de una década, las distorsiones fiscales comenzaron a aumentar después

16. Sin embargo, debería analizarse si la calidad del gasto gubernamental podría afectar el nivel de distorsiones fiscales. La literatura sobre los problemas de coordinación en la teoría económica puede sugerir que si los contribuyentes perciben que los impuestos son mal utilizados por el gobierno en gastos improductivos, estarán más dispuestos a evadir impuestos o permanecer informales en la economía (Palda, 1998). Por lo tanto, si la calidad del gasto gubernamental es cuestionable, las políticas dirigidas a reducir las distorsiones fiscales podrían fallar. 
de 2008, aparentemente por los graves niveles de corrupción en el aparato fiscal del país.

El modelo muestra que el gobierno puede ser más efectivo para aumentar la presión tributaria y dimensionar un Estado compatible con los objetivos de desarrollo, si puede reducir las distorsiones fiscales.

\section{Referencias}

Bårdsen, G. (1989). The estimation of long-run coefficients from error correction models. Oxford B Econ Stat, 54, 345-350.

Barro, R. J., y Sala-i-Martin, X. (1992). Public finance in models of economic growth. The Review of Economic Studies, 59(4), 645-661.

Blinder, A. S. (1981). Thoughts on the Laffer curve. The supply-side effects of economic policy, 81-92.

Blundell, R. (1992). Labor supply and taxation: a survey. Fiscal Studies, 13(3), 15-40.

Busato, F. y Chiarini, B. (2013). Steady state Laffer curve with the underground economy. Public Finance Review, 41(5), 608-632.

Canto, V. A., Joines, D. H., y Laffer, A. B. (2014). Foundations of supply-side economics: Theory and evidence. Cambridge, MA: Academic Press.

Dickey, D. A., y Fuller, W. A. (1979). Distribution of the estimators for autoregressive time series with a unit root. Journal of the American Statistical Association, 74, 427-431.

Engle, R.F., y Granger, C. W. J. (1987). Co-integration and error correction: representation, estimation, and testing. Econometrica, 55, 251-276.

Feige, E. L. y McGee, R. (1982a). Supply side economics and the unobserved economy: The Dutch Laffer curve. Economisch Statistische Berichten, 67(November).

Feige, E. L. y McGee, R. (1983). Sweden's Laffer curve: Taxation and the unobserved economy. The Scandinavian Journal of Economics, 85(4), 499-519.
Feige, E. L., y McGee, R. (1982b). The unobserved economy and the UK Laffer curve. The Journal of Economic Affairs, 33(1): 6-42.

Friedman, E., Johnson, S., Kaufmann, D., y Zoido-Lobaton, P. (2000). Dodging the grabbing hand: the determinants of unofficial activity in 69 countries. Journal of public economics, 76(3), 459-493.

Fuest, C., y Riedel, N. (2009). Tax evasion, tax avoidance and tax expenditures in developing countries: A review of the literature. Report prepared for the UK Department for International Development (DFID), 1-69.

Fullerton, D. (2008). Laffer curve. In Durlauf, S. N., \& Blume, L. (Eds.) The new Palgrave dictionary of economics (Vol. 6). London, UK: Palgrave Macmillan. (p. 839).

Gahvari, F. (1989). The nature of government expenditures and the shape of the Laffer curve. Journal of Public Economics, 40(2), 251.

Gërxhani, K. y Schram, A. (2006). Tax evasion and income source: a comparative experimental study. Journal Economic Psychology, 27(3), 402-422.

Gutmann, P. M., (1977). The subterranean economy. Financial Analysts Journal, 33(26), 27-34.

Heijman, W. J. M. y van Ophem, J. A. C. (2005). Willingness to pay tax: The Laffer curve revisited for 12 OECD countries. The Journal of Socio-Economics, 34(5), 714-723.

Henderson, D. (1981). Limitations of the Laffer curve as a justification for tax cuts. Cato Journal. 1(1), 45-52.

Hsing, Y. (1996). Estimating the Laffer curve and policy implications. Journal of Socio- Economics, 25(3), 395-401.

Johansen, S., (1991). Estimation and hypothesis testing of cointegration vectors in Gaussian vector autoregressive models. Econometrica, 59, 1551-1580.

Laffer, A. B. (1981). Government exactions and revenue deficiencies. Cato Journal, 1(1), 1-21.

Laffer, A. B. (2004). The Laffer curve: Past, present, and future. Backgrounder, 1765, 1-16.MacKinnon, 
J. G. (1996). Numerical distribution functions for unit root and cointegration tests. Journal of Applied Econometrics, 11, 601-618.

MacKinnon, J. G., Haug, A. A., y Michelis, L. (1999). Numerical distribution functions of likelihood ratio tests for cointegration. Journal of Applied Econometrics, 14, 563-577.

Malcomson, J. (1986). Some analytics of the Laffer curve. Journal of Public Economics, 29(3), 263.

Palda, F. (1998). Evasive ability and the efficiency cost of the underground economy. Canadian Journal of Economics, 31(5), 1118-38.

Pecorino, P. (1995). Tax rates and tax revenues in a model of growth through human capital accumulation. Journal of Monetary Economics, 36(3).

Prazmowski, P. A. (2014). Ricardian equivalence and fiscal distortions in the Dominican Republic. Empirical Economics, 41(1), 109-125.

Sanyal, A., Gang, I. N., y Goswami, O. (2000). Corruption, tax evasion and the Laffer curve. Public Choice, 105, 61-78.

Schwarz, G. E. (1978). Estimating the dimension of a model. Annals of Statistics, 6(2), 461-464.

Stuart, C. E. (1981). Swedish tax rates, labor supply, and tax revenues, Journal of Political Economy, 89(5), 1020-38.

Tanzi, V. (1977). Inflation, lags in collection, and the real value of tax revenue. IMF Staff Papers, 24, 154-167.
Torgler, B. (2005). Tax morale in Latin America, Public Choice, 122(12): 133-157.

Trabandt, M. y Uhlig, H. (2011). The Laffer curve revisited. Journal of Monetary Economics, 58(4), 305-327.

Trabandt, M. y Uhlig, H. (2013). How do Laffer curves differ across countries? In A., Alesina, and F., Giavazzi, (Ed.). Fiscal policy after the financial crisis, A National Bureau of Economic Research Conference Report, University of Chicago Press, Chapter 6: 211-249.

Transparency international (2016). Corruption Perceptions Index 2018. Disponible en: https:// www.transparency.org/news/feature/corruption_perceptions_index_2018

Transparency international (2018). Corruption Perceptions Index 2018. Disponible en: https:// www.transparency.org/news/feature/corruption_perceptions_index_2018

van Ravestein, A. y Vijlbrief, H. (1988). Welfare cost of higher tax rates: An empirical Laffer Curve for the Netherlands. De Economist, 136(2), 205-219.

Wanniski, J. (1978). Taxes, revenues, and the Laffer curve. The Public Interest, 50, 3.

Waseem, M. (2018). Taxes, informality and income shifting: Evidence from a recent Pakistani tax reform. Journal of public economics, 157, 41-77.

White, H. (1980). A Heteroskedasticity-Consistent covariance matrix and a direct test for Heteroskedasticity. Econometrica, 48, 817-838.

\section{Datos de filiación}

Peter Prazmowski. Es economista en jefe del Grupo para la Productividad y Competitividad Nacional de la República Dominicana y asesor de estrategias de E. León Jimenes. Es licenciado en Economía por la Escuela de Wharton de la Universidad de Pensilvania, con una maestría Chevening por la Escuela de Economía de Londres (LSE) y un doctorado (PhD) por la Escuela de Economía de Kingston en Londres. Ha publicado artículos científicos a nivel nacional e internacional en los campos de economía pública, teoría monetaria y macroeconomía, con énfasis en la República Dominicana. Tiene tres libros que se titulan Estabilización, Ensayos en Macroeconomía para la República Dominicana y Paises en Vias de Desarrollo con los Profesores José Sánchez-Fung y Amelia Santos Paulino, y Gazebo, por un sueño país: retos económicos que enfrenta la Repuiblica Dominicana y las acciones para superarlos con el Profesor Xavier Sala-i-Martin. 